AIRWAY PATHOLOGY

\title{
Can endobronchial biopsy analysis be recommended to discriminate between asthma and COPD in routine practice?
}

\author{
A Bourdin, I Serre, H Flamme, P Vic, D Neveu, P Aubas, P Godard, P Chanez
}

Thorax 2004;59:488-493. doi: 10.1136/thx.2003.016899

See end of article for authors' affiliations

Correspondence to: Dr P Chanez, Hôpital Arnaud de Villeneuve, 34295 Montpellier Cedex 5, France; chanez@montp. inserm.fr

Received 2 October 2003 Accepted

25 February 2004
Background: International guidelines stress the importance of accurately discriminating between asthma and chronic obstructive pulmonary disease (COPD). Although characteristic pathological features have been described for both conditions, their discriminatory power has never been systematically assessed. Methods: Endobronchial biopsy (EBB) specimens from patients with a clear clinical diagnosis of asthma and COPD (50 per group) were examined by three pathologists in a double blind manner. They were asked to propose a pathological diagnosis of either asthma or COPD and to analyse qualitatively the most frequent abnormalities reported in the literature.

Results: The sensitivity and specificity of EBB ranged from $36 \%$ to $48 \%$ and from $56 \%$ to $79 \%$, respectively. Eosinophils strongly biased the pathological diagnoses in favour of asthma, whereas their estimated prevalence was similar (11-37\% in asthma and $13-41 \%$ in COPD). Metaplasia (11-39\% in COPD, 1$18 \%$ in asthma) and epithelial inflammation (28-61\% in COPD, $11-38 \%$ in asthma) tended to be specific to COPD, whereas epithelial desquamation (80-98\% in asthma, 61-88\% in COPD) and basement membrane thickening (71-94\% in asthma, 53-88\% in COPD) tended to be associated with asthma. There was acceptable intra- and inter-observer agreement only for metaplasia and epithelial eosinophils.

Conclusions: Specific histopathological features of asthma and COPD probably exist, but current routine analysis procedures to assess EBB specimens are not sufficiently discriminatory. This might be rectified by improving pathological definitions.
R outine histopathological examination has substantially enhanced prognostic and therapeutic approaches to various diseases, particularly kidney and liver diseases and, to a lesser extent, inflammatory lung diseases. Current international guidelines ${ }^{12}$ stress the importance of clearly distinguishing between patients with asthma and chronic obstructive pulmonary disease (COPD) in order to optimise treatment and follow up. Discriminating between asthma and COPD is usually based on a combination of clinical and functional parameters. Using this approach, pathologists have gained further insight into these obstructive airflow diseases and necroscopic studies have led to classical descriptions that are available in all referenced pathology textbooks. ${ }^{3-6}$ Unfortunately, we are often confronted with complicated borderline cases ranging from smokers with asthma to patients with COPD with potentially reversible airflow impairment, for which standard clinical and functional assessments fail to give a clear diagnosis. New efficient discriminative tools are thus required to confirm the diagnosis in these borderline cases. Endobronchial biopsies (EBB) are currently performed for both diseases, but mainly for research purposes, and analysis of the results has increased the overall understanding of the underlying mechanisms. Routine analysis of EBB specimens only enables qualitative assessment of structural and inflammatory features observed in a given sample. Quantitative indices and specific immunostaining techniques ${ }^{7}$ are not currently used as they require specifically trained personnel and are too time consuming and expensive to warrant implementation in a routine setting.

The efficacy of histopathological examinations in detecting the involvement of other organs, and the reported differences in pathological features when comparing asthma and COPD, suggest that qualitative routine analysis of EBB specimens might be effective in discriminating between asthma and COPD. The lack of precision in estimating the prevalence of the most frequent pathological criteria led us to validate EBB as a diagnostic tool. To avoid an overlap between the two conditions, only patients with clearly defined asthma or COPD according to standard diagnostic criteria were studied.

\section{METHODS \\ Patients}

From 1996 to 2000 EBB was performed in six different clinical studies, approved by ethics committees, involving more than 300 patients. We selected 50 patients with well characterised mild to moderate asthma and 50 COPD patients defined according to current diagnosis guidelines. ${ }^{12}$ None of the patients had received anti-inflammatory treatment at the time of sampling. Flow-volume curves were assessed at baseline and after inhaling $400 \mu \mathrm{g}$ salbutamol via a spacer. Atopic status was assessed by skin prick tests for pneumoallergens in the Montpellier area. The concentration of methacholine provoking a fall in forced expiratory volume in 1 second $\left(\mathrm{FEV}_{1}\right)$ of $20 \%$ or more was recorded in all patients according to the published standards. ${ }^{8}$ Smoking status was checked during patient interviews. All asthma patients had a clear history of recurrent wheezing or breathlessness episodes that reversed spontaneously or after treatment, a familial history of asthma, and 15\% reversibility after bronchodilator inhalation. Patients with COPD had a clear history of smoking, chronic productive cough or sputum, no history of asthma even in their family, no reported allergic disease, and no significant reversibility after bronchodilator treatment. All patients were controlled for at least 1 month during the investigation, and none of them were receiving any treatment apart from short acting bronchodilator inhalation on demand. Bronchoscopy and 
clinical data collections were performed during the same week.

\section{EBB sampling and selection}

At least three EBBs were performed with flexible bronchoscopy under local anaesthesia on the left lower lobe with crocodile forceps according to ERS recommendations. . After fixation in neutral buffer formaldehyde $4 \%$ and embedding in paraffin, each biopsy specimen was sliced and then stained with haematoxylin-eosin. Four to six sections were fixed onto a slide for subsequent analysis. For each patient one of our team selected the best slide with respect to the presence of mucosal and submucosal layers.

\section{Randomisation and interpretation}

After randomisation a black cover with a randomly assigned number was placed on each slide. Under double blind conditions they were given to three non-specialist academic pathologists (observers A, B and C) who were asked to propose a diagnosis of asthma or COPD and to assess qualitatively the criteria reported in referenced pathology textbooks ${ }^{3-6}$ by completing a standardised form (box 1). They could add comments and note other observations, and they were free to propose a diagnosis independently of the criteria quoted. Only items valid for a diagnostic report were included in the analysis - that is, pathologists themselves decided on the threshold of significance that led them to categorise each criterion in terms of its presence or absence. The observers were asked to consider all the sections on the slide to avoid variability between sections. The pathologists were offered no additional training or published reports and were asked to conduct the diagnosis as they would in their daily practice while systematically proposing a diagnosis of asthma or COPD.

We could not calculate the number of slides required to assess the intra-observer agreement because there are no data available in the literature, so we arbitrarily used 40 randomly chosen slides for duplicate analysis.

\section{Box 1 Standardised form filled in by pathologists}

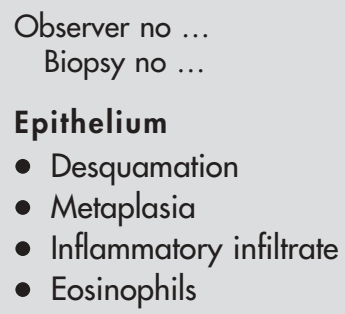

\section{Statistical analysis}

The Mann-Whitney test was used for patient comparisons. The sensitivity (biopsy specimens from asthma patients actually diagnosed as asthma), specificity (biopsy specimens from COPD patients diagnosed as COPD), and the likelihood ratio of the diagnostic value of EBB was assessed. The associations between the pathological criteria (as dichotomous variables) and pathological diagnosis were then examined. The prevalence of each criterion in asthma and COPD was estimated by maximising the log likelihood according to Walter, a statistical procedure that requires the involvement of three observers which hypothesises that there are no correlated errors and which does not lead to extrapolated results. ${ }^{10}$ Prevalences are shown as proportions with $95 \%$ confidence intervals calculated according to binomial law.

Intra- and inter-observer agreements were assessed with Cohen's kappa coefficient ${ }^{11}$ and McNemar's $\chi^{2}$ test when needed. The significance level was set at 0.05 . The computations were performed using SAS version 6.12 (SAS Institute) and Mathematica (Wolfram Research Inc, Champaign, IL, USA) software packages.

\section{RESULTS}

\section{Patient characteristics}

Patients with asthma and COPD presented with a different phenotype (table 1); 70\% of asthmatic patients were atopic and three of them were ex-smokers $(<5$ pack years $)$.

\section{EBB samples}

More than $90 \%$ of the selected slides allowed a satisfactory analysis with at least mucosal and submucosal compartments being present. Deeper structures (smooth muscle and mucosal glands) were present in more than $80 \%$ of the slides but in $45 \%$ and $32 \%$ of cases, respectively, the observers decided not to analyse them so they are not considered further in the analysis.

\section{Efficacy of EBB in the differential diagnosis of asthma and COPD}

The diagnostic value of EBB was assessed using the results of the first 100 specimens by computing the sensitivity and specificity for each observer. Two diagnoses with equal prevalences (0.5) were investigated; the results are shown in table 2 . The sensitivity and specificity were unsatisfactory for all three observers. Likelihood ratios did not statistically differ from 1, confirming that EBB has no diagnostic value. Moreover, intra- and inter-observer agreement with respect to pathological diagnosis was no longer acceptable (tables 3 and 4).

\section{Association between pathological criteria and diagnoses}

To determine why the observers obtained such low sensitivity and specificity values we first investigated the relationship between the criteria and the pathological diagnoses. Univariate analysis highlighted a very close association between the presence of eosinophils in the mucosa and submucosa $\left(\mathrm{p}<0.05\right.$ and $\mathrm{p}<10^{-9}$ for all rates, respectively) and the pathological diagnosis of asthma (table 5). We established that this close association was the main explanation for the low sensitivity and specificity since the prevalence of eosinophilic infiltration did not differ significantly between asthma and COPD when clinically diagnosed. "Epithelial eosinophils" was a reproducible criterion (tables 3 and 4) whereas "submucosal eosinophils" was not, which explains the lack of reproducibility of the pathological diagnosis. Odds ratios were considered for reproducible observers A and C. Because of the lack of reproducibility 
Table 1 Characteristics of patients with asthma or COPD

\begin{tabular}{lllr}
\hline & Asthma & COPD & p value \\
\hline Age (years) & $33(26$ to 49.8$)$ & $60(51.5$ to 66.8$)$ & $<0.0001$ \\
Sex (M/F) & $32 / 18$ & $44 / 6$ & 0.01 \\
$\mathrm{FEV}_{1}(\%)$ & $94.5(79.3$ to 100$)$ & $59.0(45.6$ to 67.8$)$ & $<0.0001$ \\
Reversibility $\beta_{2}(\%)$ & $14(11.5$ to 18$)$ & $5(0$ to 9$)$ & 0.0002 \\
Allergy (\%) & 70 & 0 & \\
Smoking (pack years) & $0(0$ to 0.75$)$ & $40(40$ to 50$)$ & \\
\hline
\end{tabular}

Values are mean ( $95 \%$ confidence intervals).

$p$ values $<0.05$ were considered statistically significant (Mann-Whitney test).

and independence of the criteria, we did not perform a logistic regression analysis.

The lack of acceptable diagnostic value of the EBB analysis prompted us to estimate the discriminative power of each criterion by computing their prevalences and reproducibility (tables 3 and 4). The intra-rate agreement was assessed using the kappa index. Observer A obtained the best results but, when all the results were considered, the intra-observer agreement was not thought to be satisfactory (table 3 ). Interobserver agreement (table 4) was weak, with the best results being obtained for metaplasia and epithelial eosinophilic infiltration.

While keeping in mind that this was a pilot study, we also looked for associations between the pathological criteria and the clinical diagnosis. By maximising the log likelihood ratio we could not identify any criterion which had a significantly different prevalence in asthma compared with COPD (fig 1). However, desquamation and thickening of the reticular basement membrane showed a trend towards a higher prevalence in asthma than in COPD, unlike metaplasia and intraepithelial inflammation. This study might not have been powerful enough to show a significant difference in the prevalence of these criteria. The number of slides required to establish significant differences (with $\alpha=0.05$ and $\beta=0.8$ ) was calculated as 61-129 slides per group $(73,61,70$ and 129 for desquamation, metaplasia, epithelial inflammation and thickening of the reticular basement membrane, respectively), whereas we only studied 50 .

\section{DISCUSSION}

The clinical and functional gold standards used to discriminate between asthma and COPD are open to criticism, but recent guidelines still advocate their use. The efficacy of the gold standard can sometimes be enhanced by including a short course of systemic steroids, ${ }^{12}$ but this would be unethical in the treatment of patients with mild to moderate controlled asthma and would substantially bias the pathological EBB results. We carefully selected patients with the two different bronchial disease entities on the basis of the most advanced clinical and functional parameters.

The qualitative analysis of EBB specimens reported in this study was neither reproducible nor discriminatory. Qualitative assessment is, however, efficient in many other diseases, indicating that certain pathological criteria are sufficiently pertinent for potential qualitative assessment applications-for example, shed $v$ metaplasic epithelium, or thickened reticular basement membrane (described as

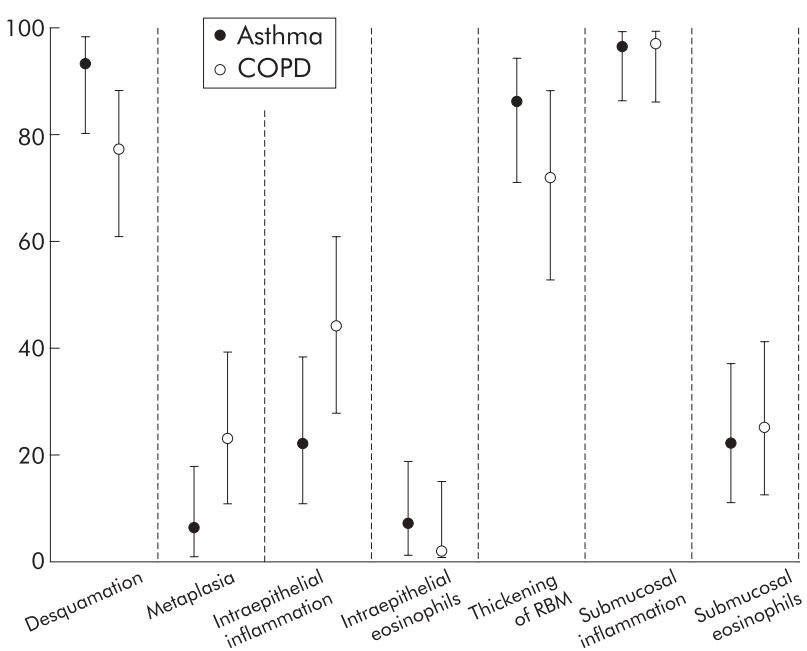

Figure 1 Comparative prevalence in asthma and COPD of all criteria studied. Data are means with 95\% confidence intervals calculated according to binomial law. No significant differences were seen between the two groups.

"pathognomonic" in previous reviews ${ }^{13}$ ). The dichotomous categorisation we used could be criticised, but it reflected the routine practice of pathologists who fixed the threshold of significance themselves. Based on a Venn's diagram, some authors ${ }^{13}$ have speculated on the possibility of a continuum of bronchial diseases ranging from COPD to asthma, with consideration of airflow reversibility and potentially overlapping conditions. In the present study we deliberately included only patients with well defined conditions, but routine pathological examinations failed to make clear distinctions between them. There are several possible reasons for this: (1) routine analysis of EBB specimens is a weaker tool than expected, indicating that "model" patients are rare and that many other factors can modify EBB analysis; (2) a more quantitative approach is needed because there is a significant overlap between patients with asthma and COPD as highlighted by the lack of a precise pathological definition; and (3) based on the findings of this study performed under real clinical conditions, asthma and COPD are relatively close entities, thus supporting the so called "Dutch hypothesis". ${ }^{14}$ Finally, our results suggest that all the criteria reported in textbooks on pathology should be considered with caution.

Table 2 Diagnostic value of endobronchial biopsies in asthma and COPD

\begin{tabular}{llll}
\hline & Sensitivity (\%) & Specificity (\%) & Likelihood ratio \\
\hline Observer A & $48(32$ to 62$)$ & $79(68$ to 91$)$ & $2.3(0.8$ to 3.8$)$ \\
Observer B & $36(22$ to 50$)$ & $63(48$ to 77$)$ & $0.97(0.4$ to 1.5$)$ \\
Observer C & $45(30$ to 60$)$ & $56(39$ to 72$)$ & $1.01(0.5$ to 1.5$)$ \\
\hline
\end{tabular}

Values are presented as mean ( $95 \%$ confidence intervals). 

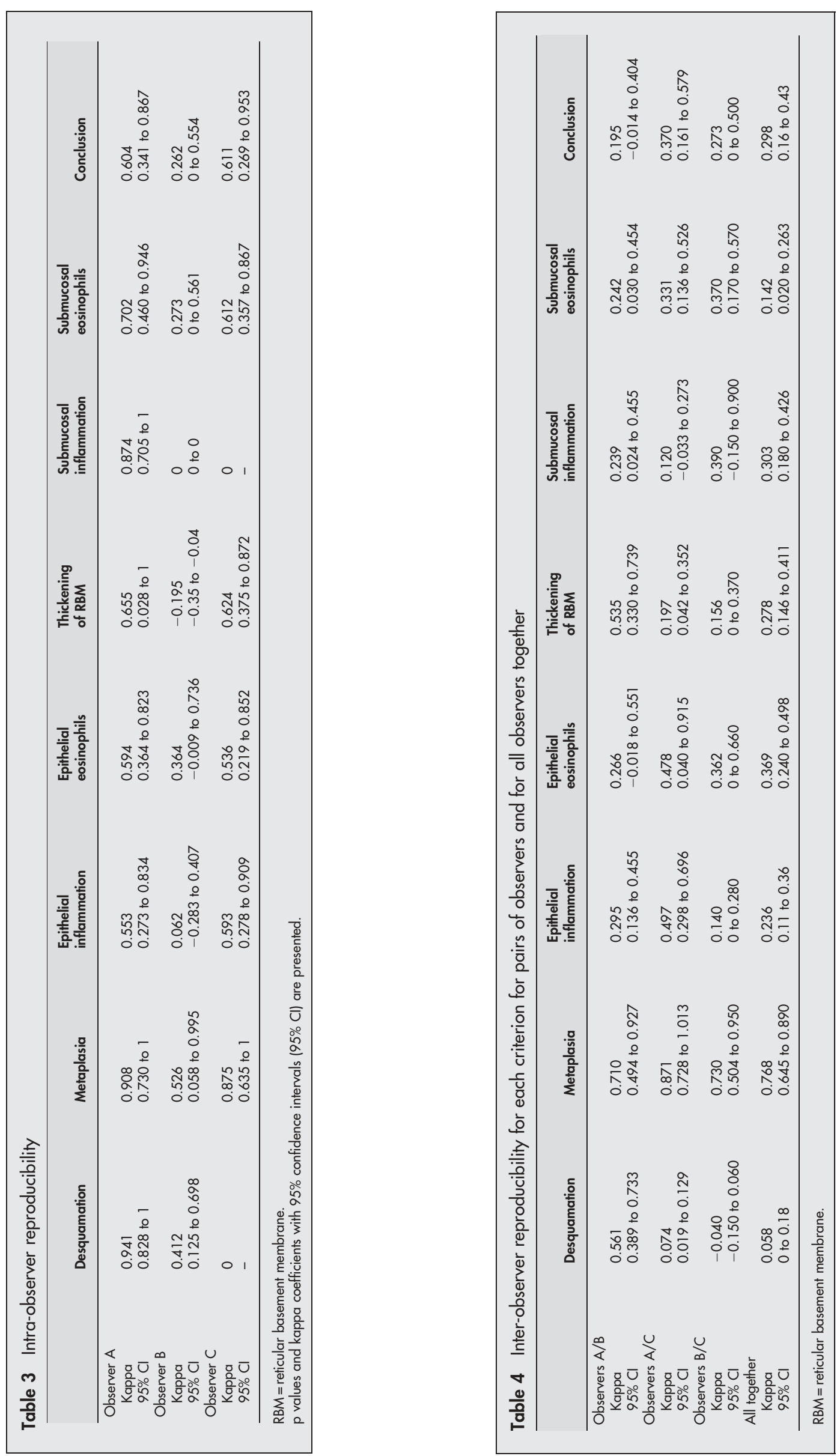

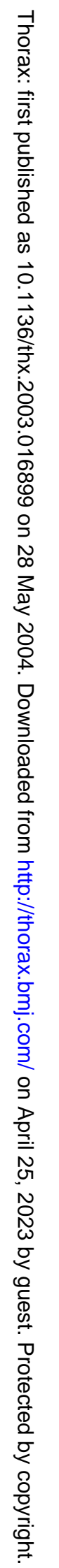




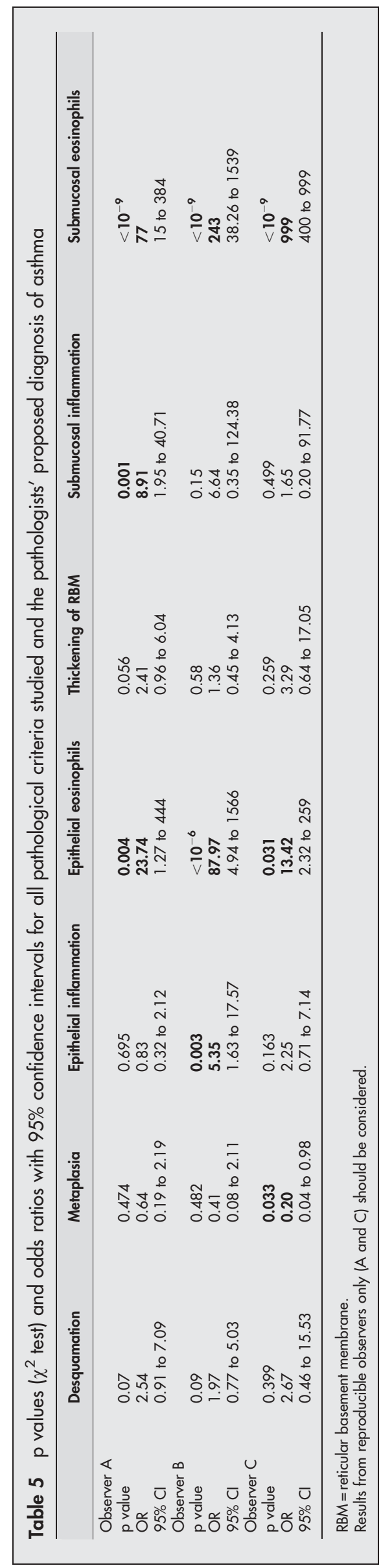

Our data clearly preclude the use of routine EBB analysis in situations where there is a possible overlap between asthma and COPD—for example, patients with asthma who smoked or COPD patients with some airflow obstruction reversibility after bronchodilation or steroid treatment. Eosinophils biased the pathological diagnosis in favour of asthma. This is not surprising since no specific immunostaining assays were performed. It has previously been reported that the presence of eosinophils cannot discriminate between asthma and COPD except when quantitative EG2+ve immunostaining is used. ${ }^{15}$ Surprisingly, the pathologists preferred to base their diagnosis on eosinophil levels which are more difficult to assess with haematoxylin-eosin staining than the thickness of the reticular basement membrane. ${ }^{7}$ This indicates a possible lack of specific training and, moreover, the need for clearer definitions since the proposals of observer A were reproducible for all criteria except the conclusion. The lack of quantitative data makes it difficult for pathologists to give a valid diagnosis, especially in the absence of a computer image analyser and when they are not ultra-specialised as in previous surveys. ${ }^{15-17}$ This indicates the small differences in the two conditions when pathological criteria are considered alone, and highlights the need to increase the specificity of the pathological definitions. The aim of this study was not to judge the ability of the pathologists to meet the gold standard but to assess the results obtained under non-specialised pathological practice conditions. It is important to note that a high degree of variability has been reported using quantitative measurements, ${ }^{18}$ and even more so in the diagnosis of cancer despite the use of standard diagnostic criteria. ${ }^{19}$ The region of interest in which EBB could eventually be used in clinical practice is in the middle of the clinical spectrum where difficult cases have inadequate responses to regular treatments. Our results confirm the absence of a diagnostic role for routine EBB in such patients.

The four pathological criteria used were quite specific to asthma or COPD, as previously reported..$^{3-6} 20$ We found that epithelial inflammation was more prevalent in COPD than in asthma, but this could be explained by the lack of quantification and risk of overestimation due to epithelial shedding observed in biopsy specimens from asthmatic patients. $^{22}$

The need for better pathological definitions led us to try to improve the validity of our results and to study the discriminative value of merging criteria into pairs. Based on the diagnostic proposals of observer A, which were the only ones that were reproducible, we obtained statistically significant discriminative criteria-desquamation plus thickened reticular basement membrane $v$ epithelial inflammation plus metaplasia successfully discriminated between asthma and COPD patients. There is now clear evidence of epithelial abnormalities in asthma leading to the often reported feature of epithelial shedding. ${ }^{23}$ These findings may enhance the efficacy of routine EBB analysis with more precise definitions, enabling specific training for pathologists to improve the diagnosis of bronchial disease.

We conclude that routine assessment of EBB specimens cannot currently be recommended to discriminate between asthma and COPD. Although there are criteria specific to these two disease entities, the biopsy specimens are difficult to analyse. Clearer pathological definitions are needed to enhance the diagnostic results.

\section{Authors' affiliations}

A Bourdin, P Godard, P Chanez, Service des Maladies Respiratoires, Hôpital Arnaud de Villeneuve, CHU de Montpellier, Montpellier, France I Serre, H Flamme, P Vic, Service d'Anatomopathologie, Hôpital Lapeyronie, CHU de Montpellier, Montpellier, France 
D Neveu, P Aubas, Département d'informatique médicale, Hôpital Arnaud de Villeneuve, CHU de Montpellier, Montpellier, France

\section{REFERENCES}

1 NHLBI. Global strategy for asthma. National Asthma Education and Prevention Program Expert Panel Report 2: Guidelines for the diagnosis and management of asthma. Publication no. 97-4051. NHLBI/WHO Workshop. Bethesda, MD: National Institutes of Health, 2002.

2 NHLBI. Global strategy for the diagnosis, management, and prevention of chronic obstructive pulmonary disease, NHLBI/WHO Workshop. Bethesda, MD: National Institutes of Health, 2001

3 Spencer H, Hasleton PS. Spencer's pathology of the lung. London: McGraw Hill, 1996.

4 Salvato G. Some histological changes in chronic bronchitis and asthma Thorax 1968;23:168-72

5 Ellis GA. The pathological anatomy of bronchial asthma. Am J Med Sci 1908;136:407-29.

6 Dunnill MS, Massarella GR, Anderson JA. A comparison of the quantitative anatomy of the bronchi in normal subjects, in status asthmaticus, in chronic bronchitis, and in emphysema. Thorax 1969;24:176-9.

7 Jeffery PK, Holgate S, Wenzel S. Methods for the assessment of endobronchial biopsies in clinical research: application to studies of pathogenesis and the effect of treatment. A consensus statement. Endobronchial biopsies in clinical research task force. Am J Respir Crit Care Med 2003; 168:S1-17

8 Sterk PJ, Fabbri LM, Quanjer PH, et al. Airway responsiveness. Standardized challenge testing with pharmacological, physical and sensitizing stimuli in adults. Eur Respir J 1993;6(Suppl 16):53-83

9 Stausz J. Pulmonary endoscopy and biopsy techniques. Eur Respir Monogr 1998;9:18-38.

10 Walter SD. Measuring the reliability of clinical data: the case for using three observers. Rev Epidemiol Santé Publ 1984;32:206-11.
11 Kramer MS, Feinstein AF. The biostatistics of concordance. Clin Pharmacol Ther 1981;29:111-23.

12 Chanez P, Vignola AM, O'Shaugnessy T, et al. Corticosteroid reversibility in COPD is related to features of asthma. Am J Respir Crit Care Med 1997; 155:1529-34.

13 Jeffery PK. Remodeling in asthma and chronic obstructive lung disease. Am J Respir Crit Care Med 2001;164:S28-38.

14 Sluiter HJ, Koeter GH, de Monchy JG, et al. The Dutch hypothesis (chronic non-specific lung disease) revisited. Eur Respir J 1991;4:478-89.

15 Lacoste JY, Bousquet J, Chanez P, et al. Eosinophilic and neutrophilic inflammation in asthma, chronic bronchitis, and COPD. J Allergy Clin Immunol 1993;92:537-48.

16 Vignola AM, Bousquet J, Chanez $P$, et al. Assessment of airway inflammation in asthma. Am J Respir Crit Care Med 1998;157:S184-7.

17 Saetta M. CD8+ T-lymphocytes in peripheral airways of smokers with COPD. Am J Respir Crit Care Med 1998;157:822-6.

18 Sont JK, Willems LN, Evertse CE, et al. Repeatability of measures of inflammatory cell number in bronchial biopsies in atopic asthma. Eur Respir J 1997:10:2602-8.

19 Layfield $\mathrm{L}$, Jones C, Rowe L, et al. Institutional review of outside cytology materials: a retrospective analysis of two institutions' experiences. Diagn Cytopathol 2002;26:45-8

20 O'Shaughnessy TC, Ansari TW, Barnes NC, et al. Reticular basement membrane thickness in moderately severe asthma and smokers' chronic bronchitis with and without airflow obstruction. Am J Respir Crit Care Med 1996; 153:A879.

21 James AL, Maxwell PS, Pearce-Pinto G, et al. The relationship of reticular basement membrane thickness to airway wall remodeling in asthma. Am J Respir Crit Care Med 2000;166:1590-5.

22 Ordonez C, Ferrando R, Hyde DM, et al. Epithelial desquamation in asthma: artifact or pathology? Am J Respir Crit Care Med 2000;162:2324-9.

23 Carayol N, Campbell A, Vachier I, et al. Modulation of cadherin and catenin expression by tumor necrosis factor- $\alpha$ and dexamethasone in human bronchial epithelial cells. Am J Respir Cell Mol Biol 2002;26:341-7. 